\title{
Summation-by-parts Operators with Minimal Dispersion Error for Accurate and Efficient Flow Calculations
}

\author{
Viktor Linders * \\ Linköping University, SE-58183 Linköping, Sweden, viktor.linders@liu.se \\ Marko Kupiainen ${ }^{\dagger}$ \\ SMHI, SE-601 76 Norrköping, Sweden, marco.kupiainen@smhi.se \\ Steven H. Frankel $\ddagger$ and Yann Delorme $\ddagger$ \\ Technion, Haifa, Israel 320003, frankel@technion.ac.il, ydelorme.ac.il \\ Jan Nordström * \\ Linköping University, SE-581 83 Linköping, Sweden, jan.nordstrom@liu.se
}

\begin{abstract}
We develop summation-by-parts operators with minimal dispersion errors both near and far from boundaries and interfaces. Such operators are superior to classical stencils for problems involving high frequency waves or multi-frequency solutions over long time intervals with a relatively coarse spatial mesh. This is demonstrated by solving the TaylorGreen vortex flow with optimised and classical operators both in a purely periodic setting as well as in the presence of numerical interfaces.
\end{abstract}

\section{Introduction and motivation}

Finite difference stencils have traditionally been constructed by optimising the formal order of accuracy with respect to the bandwidth of the scheme. The philosophy behind such stencils is that the error in the numerical solution is dominated by the truncation error, obtained by applying Taylors theorem. However, for problems involving the propagation of high frequency waves over large intervals the error may instead be dominated by inexact approximations of the dispersion relation. Such waves require small spatial increments in order to be properly resolved, which puts restrictions on the step size $\Delta x$ and thus on the efficiency of the solver.

Problems of this type are common in computational fluid dynamics, aeroacoustics, electromagnetism, metrology, elasticity and seismology. ${ }^{1-7}$ Wave properties encoded within the dispersion relation include phase velocity, group velocity, anisotropy and dissipation. It is therefore of interest to develop schemes that preserve the analytic dispersion relation of the governing equations for a wide range of spatial increments. Finite difference schemes that attempt to preserve the dispersion relation of the continuous problem have received considerable attention in the past few decades. ${ }^{8}$ It should be noted that these stencils have been primarily central and are thus not suited for problems involving boundaries or interfaces.

In this paper we approach this problem by presenting new finite difference operators on summationby-parts (SBP) form ${ }^{9-13}$ that approximate first derivatives. SBP operators in combination with weakly imposed boundary and interface conditions lead to provably stable schemes. Such operators account for the discretisation near boundaries and interfaces as well as in the interior of the computational domain. These schemes have suboptimal order of accuracy relative to the bandwidth, leaving a set of free stencil parameters that can be used to preserve the dispersion relation.

SBP operators based on the dispersion relation preserving schemes of Tam and Webb ${ }^{14}$ have previously been constructed, ${ }^{15}$ however no particular procedure was applied in order to minimise the dispersion error at

*Department of Mathematics, Computational Mathematics, Linköping University, SE-581 83 Linköping, Sweden.

${ }^{\dagger}$ Rossby Centre SMHI, SE-601 76 Norrköping, Sweden

${ }^{\ddagger}$ Faculty of Mechanical Engineering, Technion - Israel Institute of Technology, Haifa, Israel 320003 
the boundaries. In this paper we will construct SBP operators based on local stencils, ${ }^{16}$ where the dispersion error has been minimised in the $L^{\infty}$-sense. We extend this work by analogously minimising the dispersion error at the boundary of the stencil.

This paper is structured as follows: Section II introduces dispersion errors of finite difference stencils. In section III we motivate the discussion of these errors by exemplifying problems where optimised central difference stencils outperform their non-optimised counterparts. To account for boundaries and interfaces, the optimisation procedure is extended to summation-by-parts operators in section IV. In section V we look at the dispersion errors of a few examples of operators on SBP form. Relevant numerical examples are presented in section VI. Finally, conclusions are drawn in section VII.

\section{Analytic and Numerical dispersion relations}

Consider an approximation of the first derivative $u_{x}$ of some function $u(x, t)$. An equispaced finite difference stencil using $L$ points to the left and $R$ points to the right of the $l$ node can be written

$$
\left(\frac{\partial u}{\partial x}\right)_{l} \approx \frac{1}{\Delta x} \sum_{j=-L}^{R} a_{j} u_{l+j} .
$$

The normalised numerical wavenumber corresponding to this discretisation ${ }^{14}$ is given by

$$
\bar{\xi}=-i \sum_{j=-L}^{R} a_{j} \exp (i j \xi),
$$

where $\xi=\kappa \Delta x$ and $\kappa$ is the wavenumber of the Fourier transform of the analytic solution $u(x, t)$.

Noting that the smallest wavelength the scheme can resolve is $\lambda_{\min }=2 \Delta x$, we find that the largest resolvable wavenumber is $\kappa_{\max }=2 \pi / \lambda_{\min }=\pi / \Delta x$. Thus we always have $|\xi| \leq \pi$, though typically we will consider a smaller domain, $\xi \in\left[0, \xi_{\max }\right]$. Associated with the finite difference stencil is thus a dispersion error defined as $E(\xi, \mathbf{a})=\xi-\bar{\xi}$, where a is a vector containing the stencil parameters $a_{j}$. The typical task is to choose $\mathbf{a}$ in such a way that $E$ is minimal in the sense of a suitable norm, under some accuracy or compatibility requirements on the resulting stencil. We shall throughout the remainder consider minimisation in the $L^{\infty}$-norm on the interval $\xi \in\left[0, \xi_{\max }\right]$.

\section{Minimal dispersion error in the interior domain}

In previous work ${ }^{16}$ it was shown how to construct central finite difference stencils that minimise the dispersion error $E(\xi, \mathbf{a})$ in the $L^{\infty}$-sense for $\xi \in\left[0, \xi_{\max }\right]$, given a certain order of accuracy and stencil width. We will use these stencils (denoted Remez) in the interior solution domains, i.e. away from any boundaries or interfaces. In Figure 1 examples of Remez stencils are compared with other stencils presented in the literature. ${ }^{14,17,18}$ Here, schemes denoted Classical are those of maximum order of accuracy with respect to the bandwidth. The numbers in brackets represent the formal order of accuracy and the bandwidth of the stencil respectively.

The error curves shown in Figure 1 are obtained from a thoroughly polychromatic Gaussian pulse solution to the periodic advection problem. ${ }^{16}$ Clearly the Remez $(2,9)$ stencil is the best performer for long times. Note that $\operatorname{Remez}(2,7)$ is the best stencil among those that are seven points wide. In fact, it even performs better than the Classical $(8,9)$ stencil despite having lower accuracy and utilises less bandwidth.

Figure 2 shows snapshots of vorticity at $t=0.95$ for the periodic double shear layer problem with Reynolds number $R_{e}=10^{4}$, solved on two different grids. These results were obtained using a 6th order Remez stencil and optimised for $\xi \in[0, \pi / 2]$, to spatially discretise the incompressible Navier-Stokes equations. ${ }^{19}$ The coarser grid results $(64 \times 64$ points $)$ show spurious vortices in the braids of the shear layer, consistent with previously published under-resolved results for the same problem. The finer grid results $(128 \times 128$ points $)$ are in excellent qualitative agreement with published data on much finer grids. ${ }^{20-22}$ Hence, the new central schemes appear to offer high-resolution for sharp gradients on relatively coarse grids and these results suggest significant potential for high-resolution large eddy simulation of turbulent flows. 


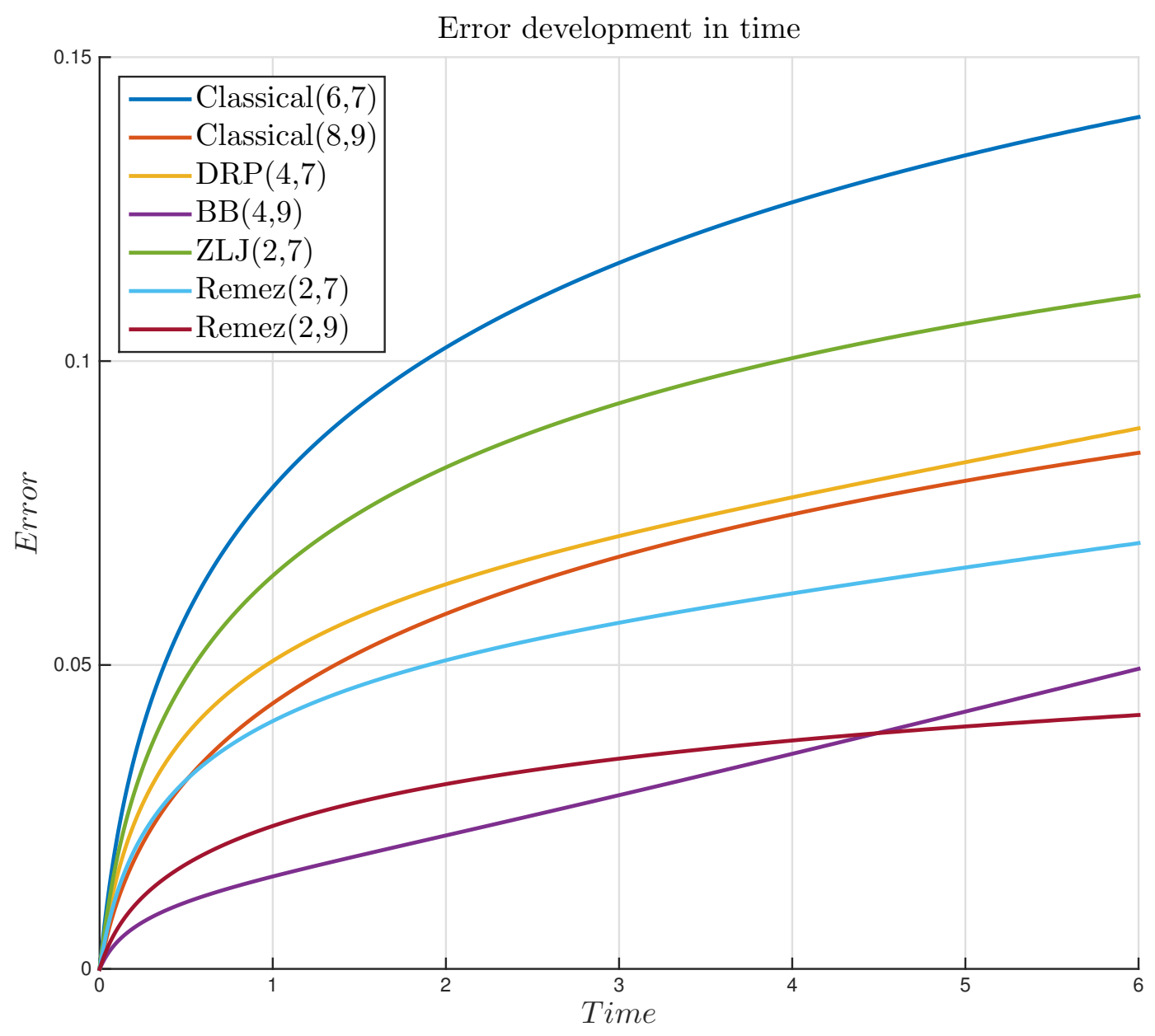

Figure 1: $L^{2}$-errors of a polychromatic solution of the advection equation.

\section{Optimised SBP operators}

Motivated by the previous results we wish to address the issue of constructing stencils with minimal dispersion error near boundaries and interfaces using summation-by-parts operators (SBP). SBP operators ${ }^{9-12,23}$ augmented with Simultaneous Approximation Terms (SAT) ${ }^{24}$ that weakly enforce boundary conditions (the SBP-SAT technique) allow for stable and high order discretisations of systems of partial differential equations if well posed boundary conditions are available.

A summation-by-parts operator $D=P^{-1} Q$ may be defined by the properties

1. $\frac{\partial u}{\partial x}=D \mathbf{u}+\mathcal{O}\left(\Delta x^{m+1}\right)$,

2. $P=P^{T}>0$,

3. $Q+Q^{T}=\operatorname{diag}(-1,0, \ldots, 0,1)$,

where $P$ and $Q$ are matrices of dimension $(N+1) \times(N+1)$, and $\mathbf{u}$ is the projection of the function $u(x, t)$ onto the grid.

Remark 1. There are more general formulations than 1,2,3 above that extend the definition of summationby-parts operators to non-cartesian grids. ${ }^{25-27}$ 


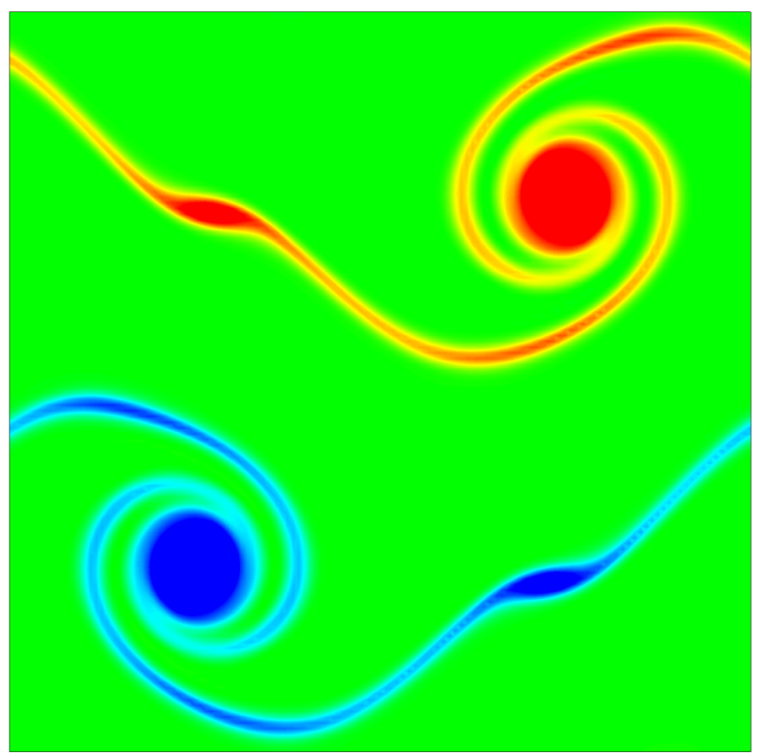

(a) Resolution: $64 \times 64$.

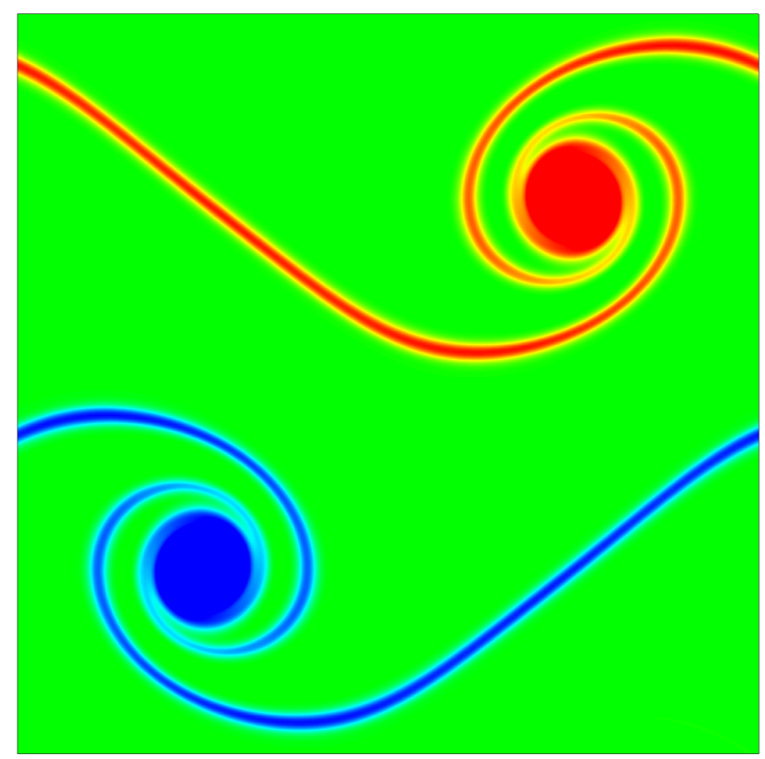

(b) Resolution: $128 \times 128$.

Figure 2: Solutions of the periodic double shear layer problem at time $t=0.95$ with Reynolds number $R_{e}=10^{4}$.

For stability reasons ${ }^{28}$ we will here limit our analysis to the case when $P$ is a diagonal matrix of the form

$$
P=\Delta x \operatorname{diag}\left(p_{1}, \ldots, p_{r}, 1, \ldots, 1, p_{r}, \ldots, p_{1}\right) .
$$

As an example of the typical structure of $Q$ we consider

$$
Q=\left(\begin{array}{ccccccc}
-1 / 2 & q_{11} & q_{12} & q_{13} & & & \\
-q_{11} & 0 & q_{21} & q_{22} & & & \\
-q_{12} & -q_{21} & 0 & q_{31} & a_{2} & & \\
-q_{13} & -q_{22} & -q_{31} & 0 & a_{1} & a_{2} & \\
& & -a_{2} & -a_{1} & 0 & a_{1} & a_{2} \\
& & & & & \ddots &
\end{array}\right)
$$

where $\mathbf{a}=\left(a_{1}, a_{2}\right)$ is the vector of coefficients of a known central difference stencil (such as a Remez stencil), and $q_{i j}$ are stencil coefficients to be determined. The index $i$ corresponds to the row where $q_{i j}$ first appears. The index $j$ corresponds to its location with respect to the main diagonal. Thus, $Q$ consists of a given interior set of central difference stencils augmented with unknown boundary blocks of size $r \times r$ to account for the discretisation near domain boundaries. We want to choose the coefficients in the boundary blocks such that each row is associated with an as small dispersion error as possible, without destroying the accuracy of the resulting scheme.

Let $\mathbf{q}$ and $\mathbf{p}$ be vectors containing the boundary coefficients of $Q$ and $P$ respectively. Each row of the boundary block defines a finite difference stencil of the form (1) and therefore has an associated dispersion relation of the form (2), and consequently a dispersion error. In what follows we will restrict our attention to the real part of the dispersion errors as the imaginary part affects the amplitude of the solution but not the dispersion properties. Thus, with a slight abuse of notation, we let the real part of the dispersion error at the $j$ th row of the boundary of $D$ be given by

$$
E_{j}(\xi, \mathbf{q}, \mathbf{p})=\xi-\bar{\xi}_{j}(\xi, \mathbf{q}, \mathbf{p}),
$$

where $\bar{\xi}_{j}(\xi, \mathbf{q}, \mathbf{p})$ is the real part of the numerical wavenumber of the stencil at the $j$ th row of $D$. 
We define the index set $\mathcal{J}_{r}=\{1,2, \ldots, r\}$ and formulate our problem as follows:

$$
\begin{array}{rrr}
\text { Find } & \min _{\mathbf{q}, \mathbf{p}}\left[\max _{j \in \mathcal{J}_{r}}\left\|E_{j}(\xi, \mathbf{q}, \mathbf{p})\right\|_{\infty}\right] \\
& \text { subject to } \\
\text { and } & A_{0} \mathbf{p}=\mathbf{b}_{\mathbf{0}} \\
A_{1} \mathbf{q} & =\mathbf{b}_{\mathbf{1}} .
\end{array}
$$

Here $A_{0}$ and $\mathbf{b}_{0}$ are obtained by requiring that $Q x^{j}=j P x^{j-1}$ for $j=0, \ldots, m$, which results in a scheme of order $\mathcal{O}\left(\Delta x^{m}\right)$ near boundaries. Further, $A_{1}$ ensures that $P$ is positive definite in accordance with the definition of an SBP operator. The problem (3) is tackled by rewriting it as a quasiconvex programming problem $^{29}$ and solved accordingly.

\section{Examples of SBP operators}

Stencils on SBP form may be defined through the triplet $(m, n, r)$, where $m$ is the order of accuracy, $n$ is the number of free parameters in the interior stencil and $r$ is the dimension of the boundary block. The cost of using a given SBP operator depends on its sparsity, which in its turn depends on $r$ and the bandwidth of the inner stencil, $2(m+n)+1$. We will henceforth primarily consider two examples of SBP operators, each with $r=8$ and $m+n=4$. The coefficients of these are found in Appendix A. The first of these is a "classical" (in the sense of non-optimised) SBP operator with $(m, n, r)=(4,0,8)$. We will refer to this operator as $\operatorname{SBP}(4,0,8)$.

The second operator have $(m, n, r)=(2,2,8)$, where all free parameters resulting from the reduced accuracy are used to minimise the dispersion error in the domain $\xi \in[0, \pi / 3]$. We will refer to this operator as $\operatorname{SBP}(2,2,8)$. The dispersion error at each row of the two operators are shown in Figure 3. The dispersion errors of $\operatorname{SBP}(2,2,8)$ are reduced by more than a magnitude for the higher wavenumbers compared to the $\operatorname{SBP}(4,0,8)$ operator.

\section{Numerical examples}

In what follows we will illustrate the performance of the optimised SBP operators through two numerical examples. The first is a narrow Gaussian pulse solution to the one-dimensional advection equation. This simple illustration exemplifies the use of optimised operators for solutions with high frequency spectral content. The second example is the Taylor-Green vortex, which illustrates how optimised operators regain much of the resolving capacity of central differences for periodic problems.

\section{A. Gaussian pulse}

We consider a polychromatic solution to the advection equation

$$
u_{t}+u_{x}=0, \quad-3.5 \leq x \leq 3.5, \quad t \geq 0
$$

with exact solution

$$
u(x, t)=2 \exp \left(-3200(x-t+3)^{2}\right) .
$$

This pulse is narrow and thus its Fourier transform is wide resulting in a significant contribution from a broad range of wavenumbers.

The spatial derivative is approximated by the $\operatorname{SBP}(4,0,8)$ and $\operatorname{SBP}(2,2,8)$ operators described previously. Additionally, another non-optimised SBP operator with $(m, n, r)=(2,0,4)$ is used to compare the performance of $\operatorname{SBP}(2,2,8)$ with a scheme of the same order of accuracy. This operator will be denoted $\operatorname{SBP}(2,0,4)$. We set $\Delta x=1 / 120$ and integrate in time using the classical fourth order Runge-Kutta scheme with time step $\Delta t=10^{-3}$ so the contribution from the temporal discretisation is small. To emphasise the effect of the boundary blocks in the SBP operators we add a numerical interface at $x=0$.

The exact and numerical solutions are shown in Figure 4, where we have separated the pulses vertically to aid the visualisation. Clearly, all numerical solutions quickly disperse into a train of pulses of decaying amplitude trailing behind the main peak. The pulse trains are a consequence of the inability of the stencils to resolve high frequency Fourier modes properly. Note that the trail behind the $\operatorname{SBP}(2,2,8)$ operator is smaller than the others as is expected from the smaller dispersion errors shown in Figure 3. 


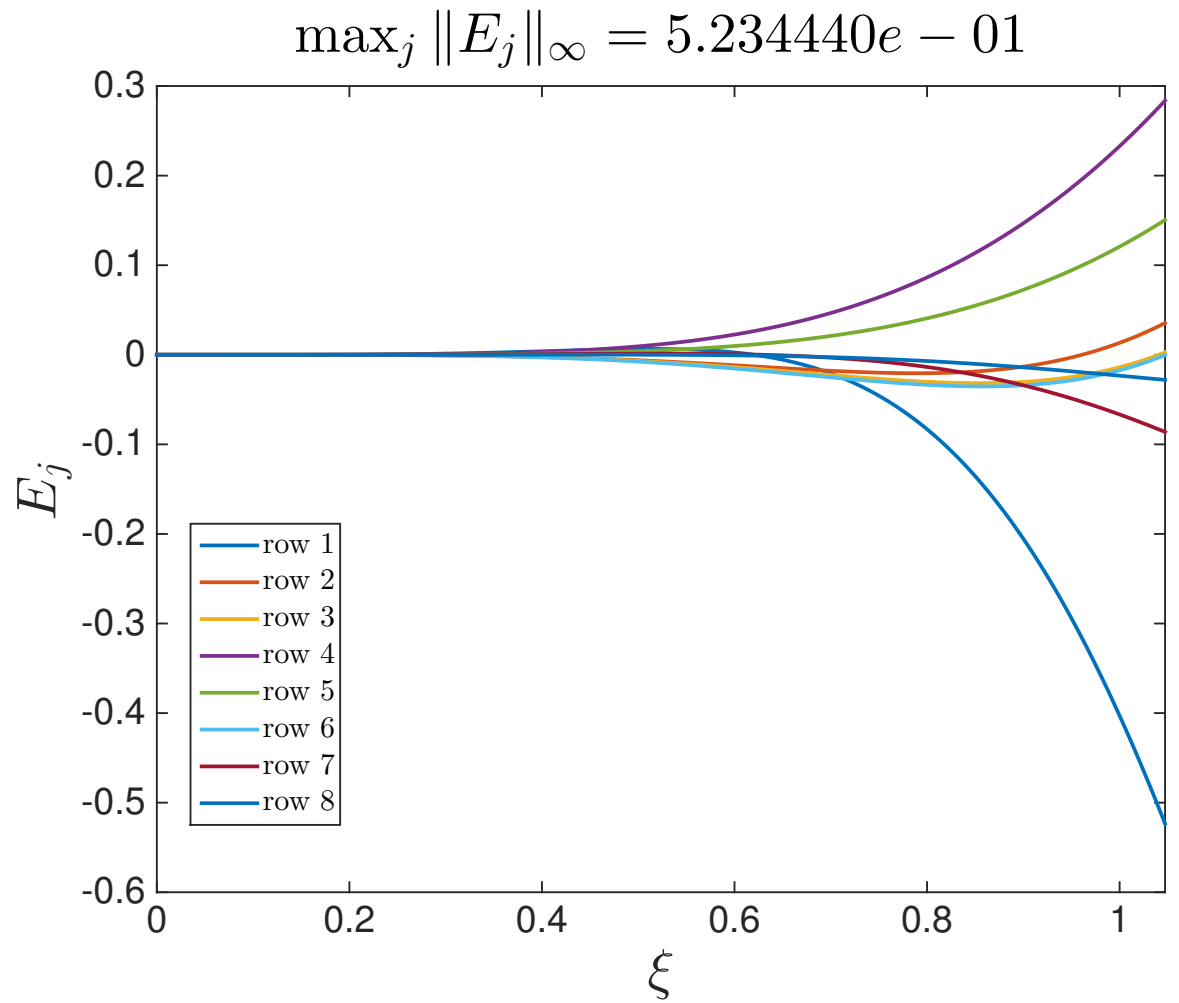

(a) $\operatorname{SBP}(4,0,8)$

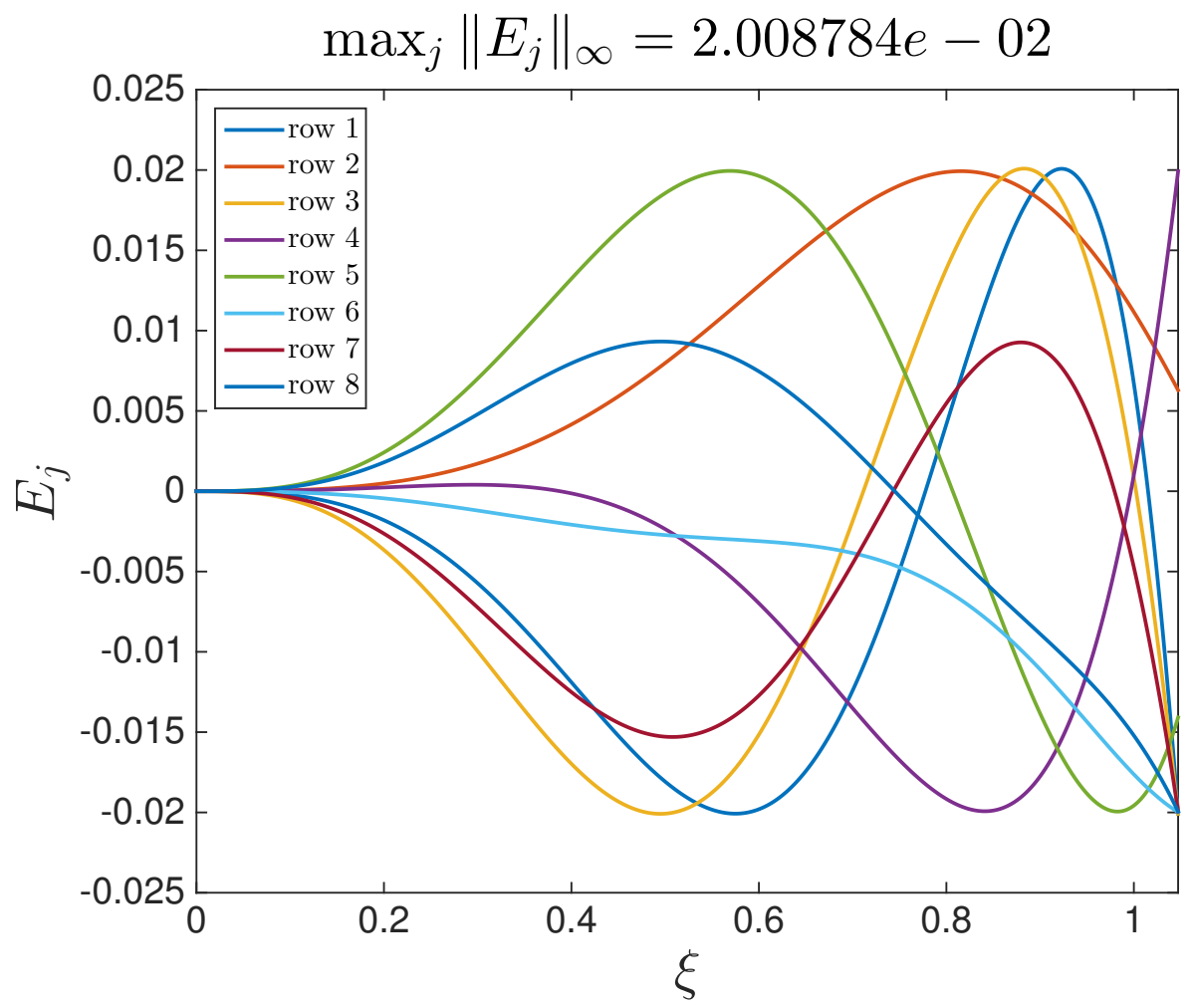

(b) $\operatorname{SBP}(2,2,8)$

Figure 3: Dispersion errors at the boundary of two different SBP operators. 


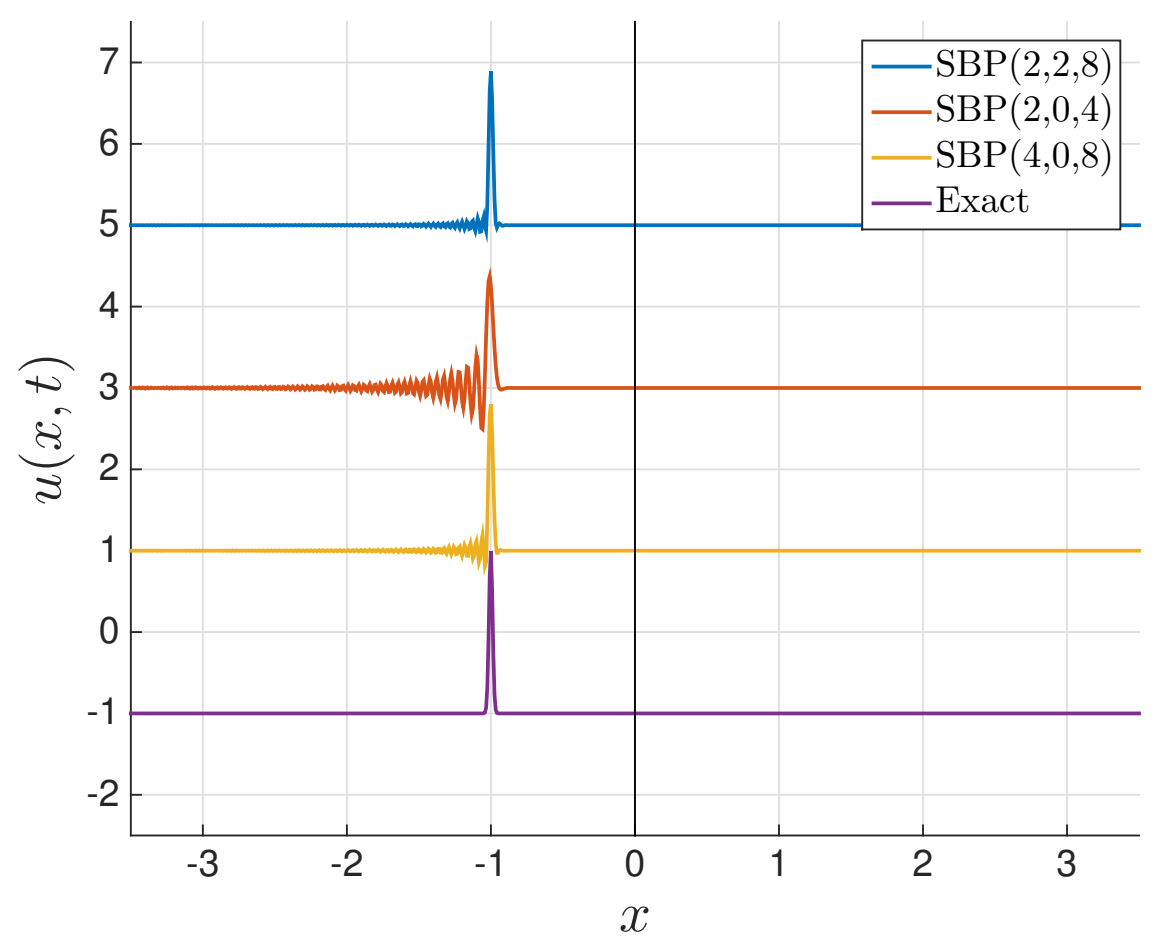

(a) Solutions prior to the interface.

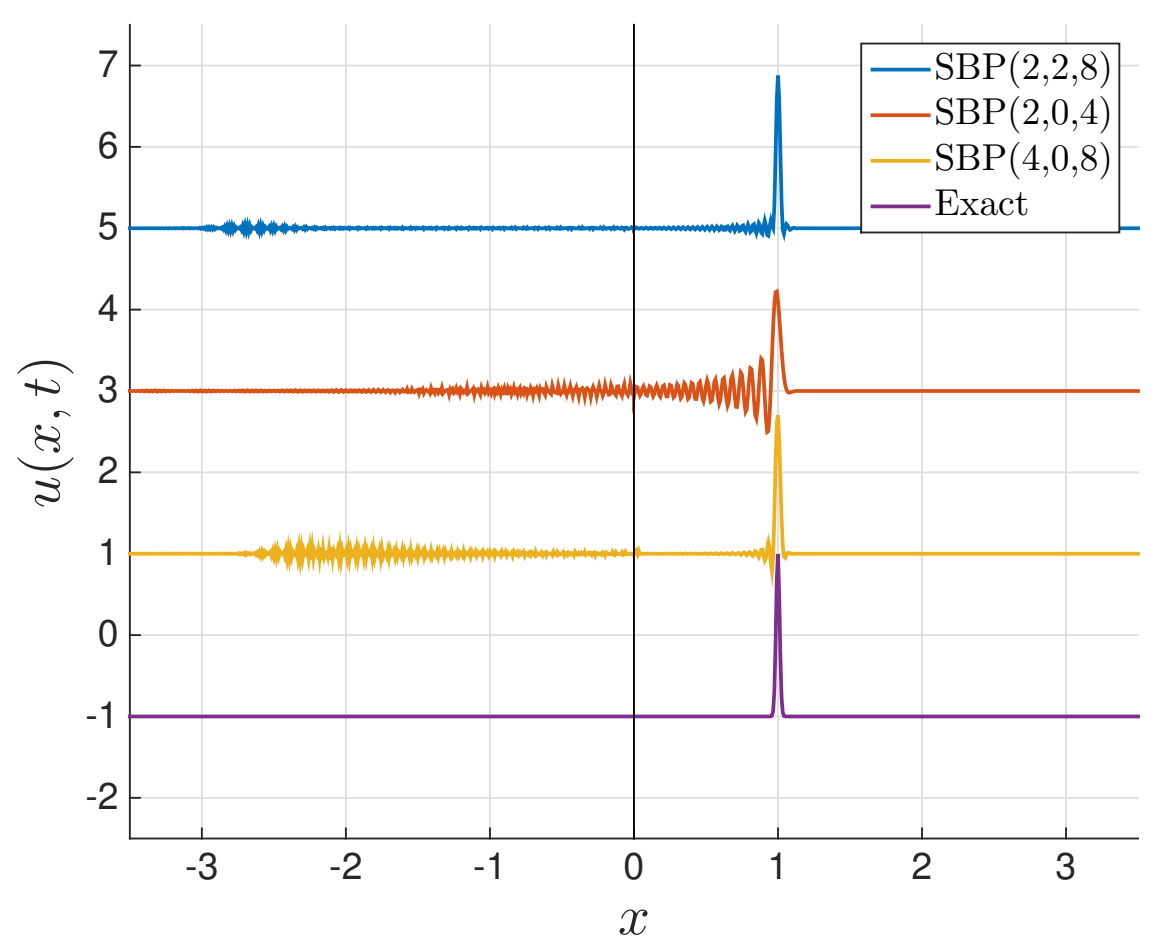

(b) Solutions after the interface.

Figure 4: Numerical solutions to the polychromatic advection problem before and after the pulses pass through the numerical interface. 


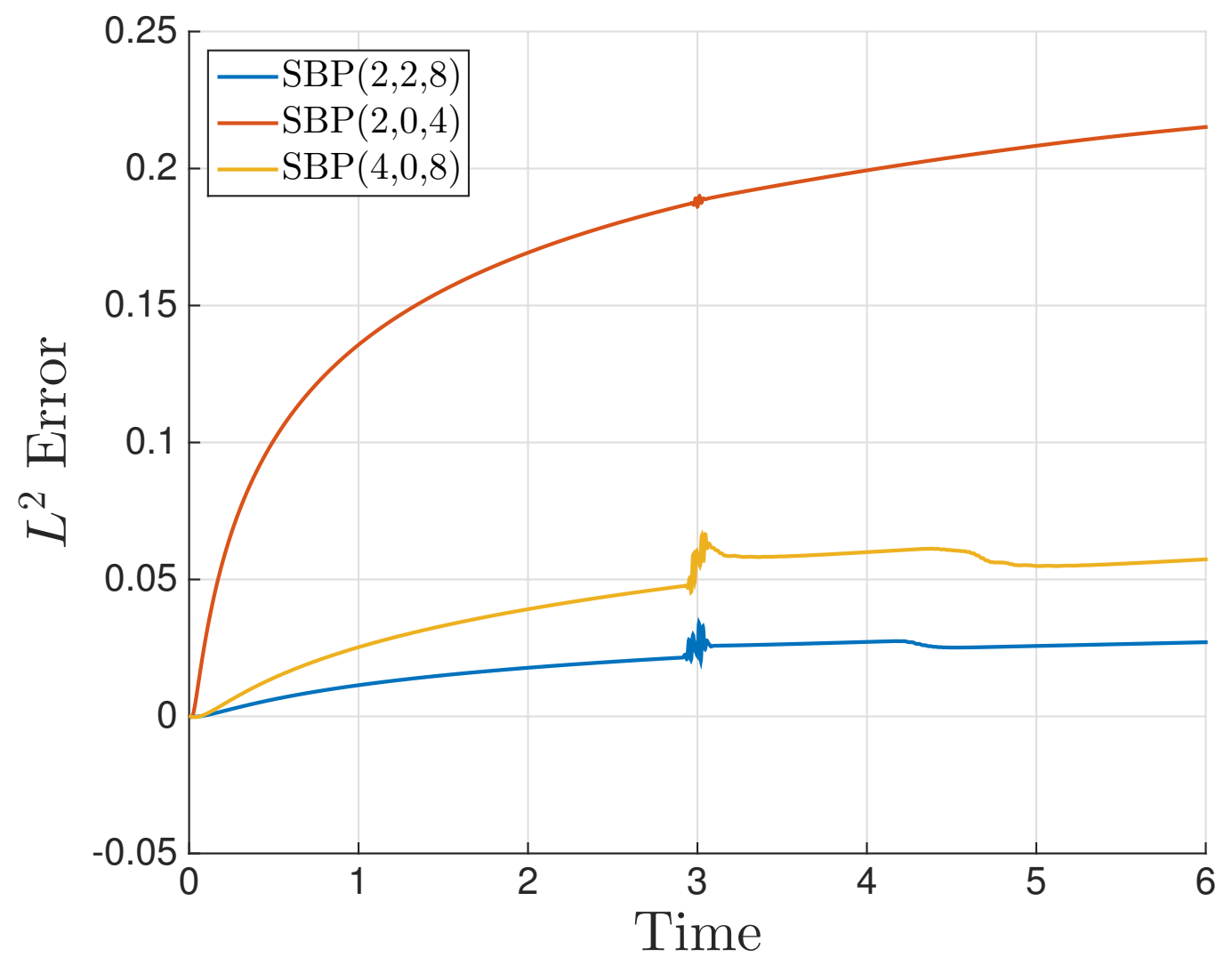

Figure 5: Error development.

Numerical reflections of unresolved Fourier modes are seen after the pulses pass through the interface. Clearly the reflection is smaller when using the $\operatorname{SBP}(2,2,8)$ operator compared to the non-optimised SBP stencils. Note also that the spurious reflections propagate out of the domain faster by the optimised stencil than by the classical SBP operators. This results from the fact that the classical stencils underrepresent the wave speed of high frequency modes, ${ }^{16}$ whereas this effect is significantly reduced after minimising the dispersion error.

The $L^{2}$ error as a function of time is plotted in Figure 5. The disturbance around $t=3$ is due to interactions with the numerical interface. Again it is seen that the optimised $\operatorname{SBP}(2,2,8)$ stencil is the better performer both at and away from boundaries and interfaces. Clearly the $\operatorname{SBP}(2,0,4)$ operator is significantly worse than $\operatorname{SBP}(2,2,8)$ despite the schemes having the same formal order of accuracy.

\section{B. Taylor-Green vortex}

The Taylor-Green vortex (TGV) flow in a three dimensional periodic box is a model for the non-linear transfer of kinetic energy among eddies with a large range of sizes. Velocity shear generates kinetic energy, which is dissipated by the viscosity at smaller scales. Thus, the TGV provides a model problem for the evolution of a turbulent flow and the energy transfer to successively smaller spatial scales. The governing equations for the TGV will here be the 3D compressible Navier-Stokes equations with constant physical properties and a low Mach number, $M_{0}=0.1$. We assume that the fluid is a perfect gas at Reynolds number $R e=1600$ with heat capacity ratio $\gamma=1.4$ and Prandtl number $\operatorname{Pr}=0.71$. The initial temperature field is assumed to be constant. 


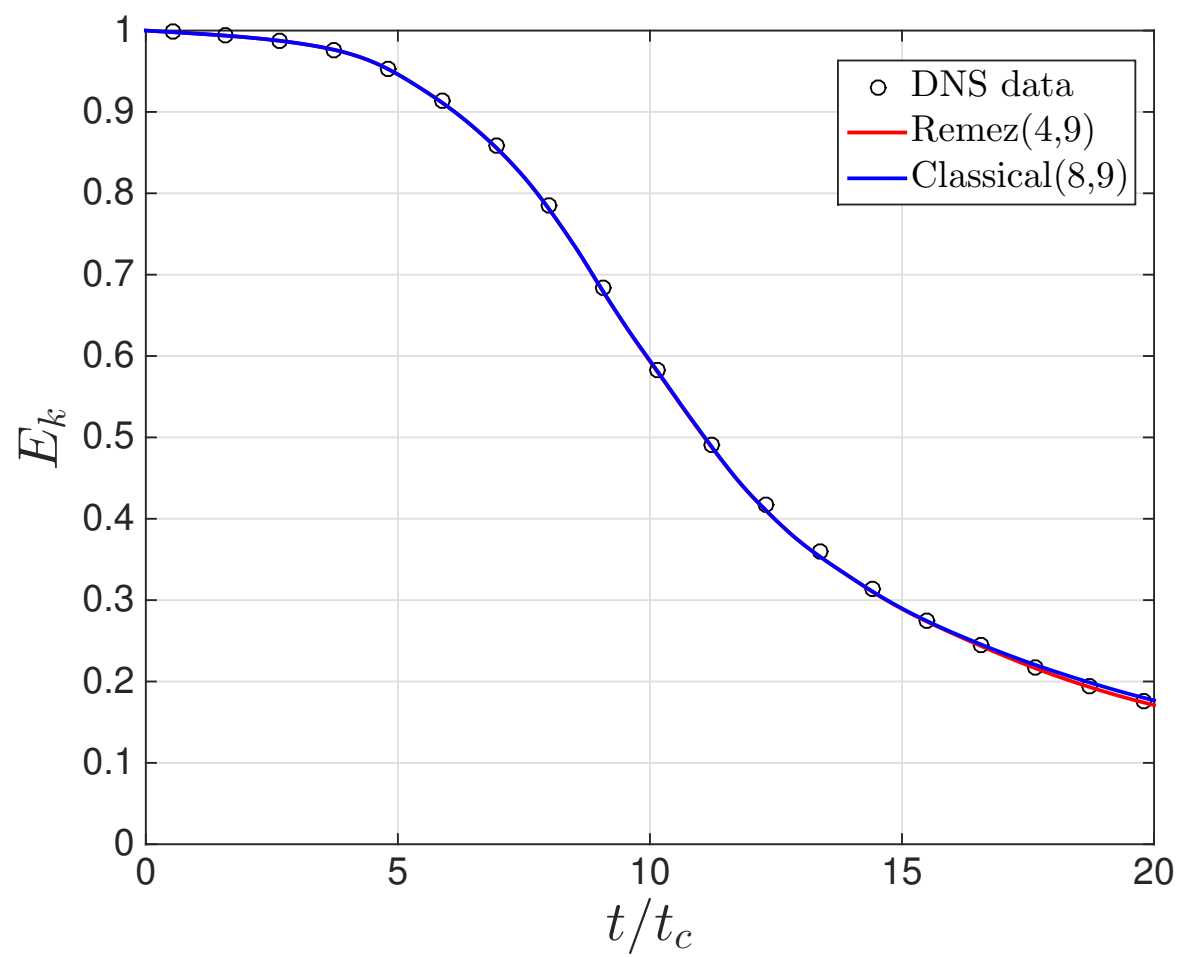

(a) Purely periodic problem.

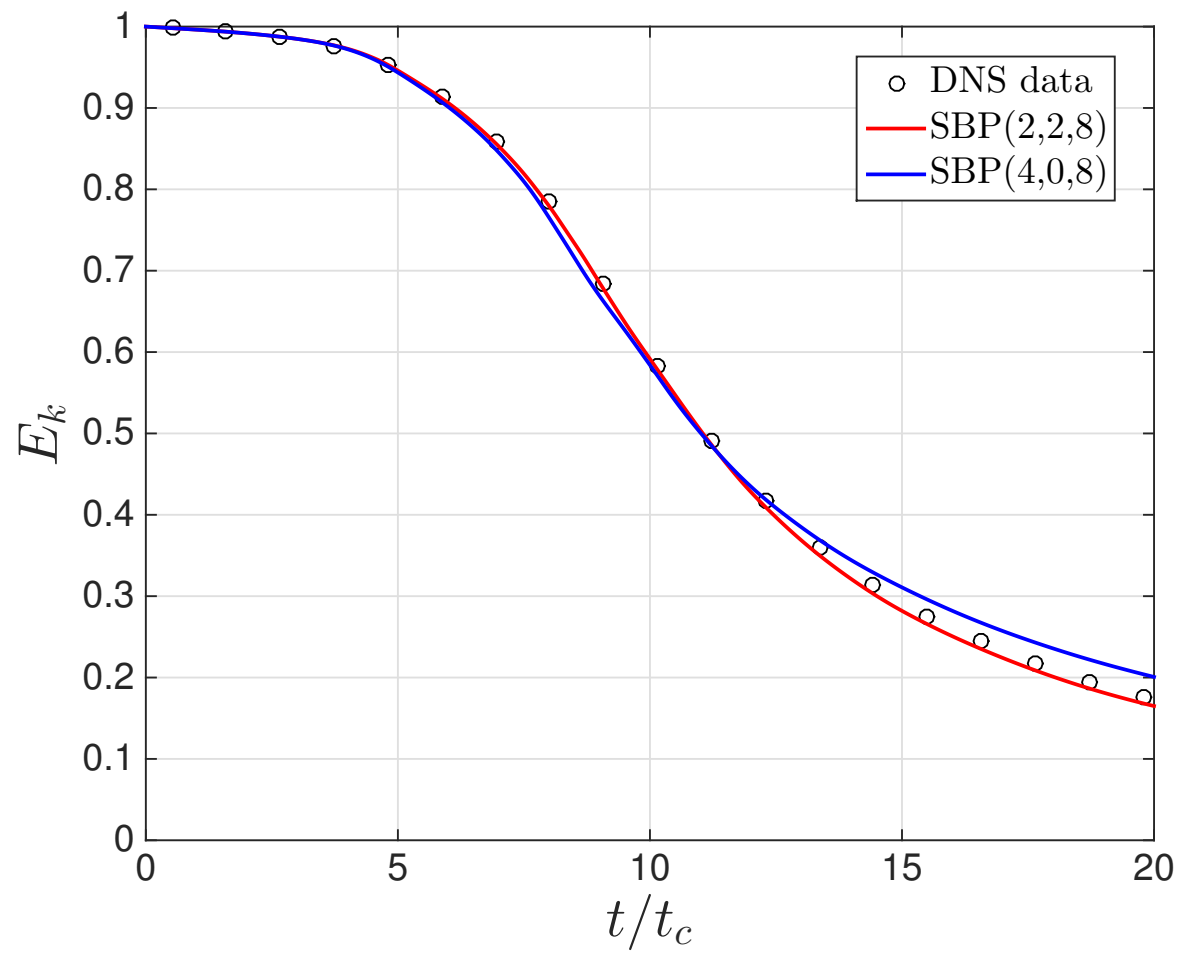

(b) Interface problem.

Figure 6: Kinetic energy evolution for the purely periodic and the interface problems. 
The initial conditions are given by

$$
\begin{aligned}
u & =V_{0} \sin \left(\frac{x}{L}\right) \cos \left(\frac{y}{L}\right) \cos \left(\frac{z}{L}\right) \\
v & =-V_{0} \cos \left(\frac{x}{L}\right) \sin \left(\frac{y}{L}\right) \cos \left(\frac{z}{L}\right) \\
w & =0 \\
p & =1+\frac{V_{0}^{2}}{16}\left(\cos \left(\frac{2 x}{L}\right)+\cos \left(\frac{2 y}{L}\right)\right)\left(\cos \left(\frac{2 y}{L}\right)+2\right),
\end{aligned}
$$

where $u, v, w$ and $p$ represent the flow field in the $x, y$ and $z$ directions, and the pressure respectively. Here $V_{0}=0.1$ and $L=2 \pi$ is the dimension of the periodic box. The duration of the flow is taken to be $20 t_{c}$ where $t_{c}$ is the characteristic convective time, $t_{c}=L / V_{0}$.

The problem is solved using Essense; a general purpose code that uses high-order temporal and spatial discretisation schemes to simulate the compressible Navier-Stokes equations. The code is written entirely in Fortran 90 and utilises Message Passing Interface (MPI) libraries for parallelisation. The code uses the fourth order explicit Runge-Kutta time stepping scheme for time-integration. SBP-stencils are used for the spatial discretisation and SAT imposes the boundary conditions weakly. The SBP stencil is of arbitrary size and the spatial discretisation can be varied by simply changing the width of the stencil and the coefficients.

As a measure of the quality of the scheme we compute the kinetic energy of the solution and compare it to data from a direct numerical simulation obtained using a dealiased pseudo-spectral code, free from both numerical dissipation and dispersion errors in space, and used on a $512^{3}$ point grid. ${ }^{30,31}$ In our simulation we integrate the problem on a $128^{3}$ point grid at a Courant number of 0.1 .

We consider two possible scenarios: The first is a purely periodic problem for which the central difference stencils at the interior of the SBP operators are utilised everywhere. For consistency with Figure 1 we refer to the interior stencil of the $\operatorname{SBP}(4,0,8)$ operator as Classical $(8,9)$. In this case, without boundary and interface blocks, Classical $(8,9)$ constitutes an 8th order accurate scheme with a bandwidth of 9 points. Similarly, the interior of the $\operatorname{SBP}(2,2,8)$ operator will be referred to as $\operatorname{Remez}(4,9)$, which is 4 th order accurate and also utilises a bandwidth of 9 points.

For most realistic applications some geometry will be present in the problem for which physical and/or numerical interfaces must be accounted. Here, the SBP operators with boundary blocks are used. In our second scenario we therefore introduce numerical interfaces at the edges of the box, enforcing periodic boundary conditions using the weak SAT formulation. As previously stated, this introduces boundary blocks in the SBP operators and reduces the formal order of accuracy of $\operatorname{SBP}(4,0,8)$ to $\mathcal{O}\left(\Delta x^{4}\right)$ and to $\mathcal{O}\left(\Delta x^{2}\right)$ for $\operatorname{SBP}(2,2,8)$ with corresponding schemes of order 5 and 3 respectively. Additionally, the resolving capacities at the boundaries are reduced.

In Figure 6 the time evolution of the kinetic energy is plotted for the two scenarios. In the periodic case both the 8 th order Classical $(8,9)$ operator and the optimised Remez $(4,9)$ operator resolve high wavenumbers sufficiently well to capture the energy cascades from larger to smaller scales accurately at all times (details about the dispersion properties of central difference schemes have been presented in previous work ${ }^{16}$ ).

With the added interface the classical $\operatorname{SBP}(4,0,8)$ fails to capture the energy decay properly, both in the transition to turbulence at $t / t_{c} \sim 8$ and at the end of the simulation. The optimised $\operatorname{SBP}(2,2,8)$ operator still captures the energy transition accurately at all times despite the scheme being of lower order. Thus, the superior resolving capacity of higher wavenumbers resulting from minimising the dispersion errors at the boundary of the SBP operator outweighs the reduced formal order of accuracy.

\section{Conclusion}

An inaccurate representation of the analytic dispersion relation by finite difference stencils may for problems involving high wavenumbers or large time scales be the dominant source of error. To remedy this, a variety of central difference schemes have been presented in the literature that preserves the dispersion relation of the governing equations. In this paper we have developed summation-by-parts operators that extend this dispersion relation preservation to stencils near boundaries and interfaces, thus allowing for a much broader range of problems to be solved with high spectral resolution.

Comparisons of an optimised and a non-optimised SBP operator of the same order has revealed that significant error reductions can be obtained for propagating solutions with high frequency content. Further 
comparisons with a higher order operator has shown that maximising the formal order of accuracy with respect to the bandwidth of the stencil is inferior to minimising the dispersion error, both for problems involving high frequencies and for long time simulations of turbulent flows.

Finally it has been demonstrated that an optimised SBP operator gives excellent agreement with data obtained on a much finer grid for the Taylor-Green vortex even in the presence of an artificial interface. This suggests that optimised SBP operators are promising tools also for turbulence simulations governed by initial boundary value problems with a physical geometry. Further research into this approach will be an interesting topic for a future paper.

\section{A. Stencil coefficients}

\section{$\operatorname{SBP}(4,0,8)$}

\begin{tabular}{|c|c|c|c|c|c|}
\hline & $Q$ interior & & $Q$ boundary & & $P$ boundary \\
\hline$a_{1}=$ & 0.800000000000000 & $q_{12}=$ & 0.659635717828799 & $p_{1}=$ & 0.294890676177879 \\
\hline$a_{2}=$ & -0.20000000000000 & $q_{13}=$ & 0.003636792564243 & $p_{2}=$ & 1.525720623897707 \\
\hline$a_{3}=$ & 0.038095238095238 & $q_{14}=$ & -0.251896871981815 & $p_{3}=$ & 0.257452876984127 \\
\hline$a_{4}=$ & -0.003571428571429 & $q_{15}=$ & 0.019621480536656 & $p_{4}=$ & 1.798113701499118 \\
\hline & & $q_{16}=$ & 0.104823702050265 & $p_{5}=$ & 0.412708057760141 \\
\hline & & $q_{17}=$ & -0.031813303755144 & $p_{6}=$ & 1.278484623015873 \\
\hline & & $q_{18}=$ & -0.004007517243009 & $p_{7}=$ & 0.923295579805997 \\
\hline & & $q_{23}=$ & 0.099349785052917 & $p_{8}=$ & 1.009333860859158 \\
\hline & & $q_{24}=$ & 0.848982308201037 & & \\
\hline & & $q_{25}=$ & -0.119397987397090 & & \\
\hline & & $q_{26}=$ & -0.268311094576721 & & \\
\hline & & $q_{27}=$ & 0.093867394179891 & & \\
\hline & & $q_{28}=$ & 0.005145312368774 & & \\
\hline & & $q_{34}=$ & -0.034454778439160 & & \\
\hline & & $q_{35}=$ & 0.235388351521182 & & \\
\hline & & $q_{36}=$ & -0.091354910714283 & & \\
\hline & & $q_{37}=$ & -0.021532738095240 & & \\
\hline & & $q_{38}=$ & 0.014940653344673 & & \\
\hline & & $q_{45}=$ & 0.026881820436502 & & \\
\hline & & $q_{46}=$ & 0.726309110449733 & & \\
\hline & & $q_{47}=$ & -0.173035530570249 & & \\
\hline & & $q_{48}=$ & -0.017524742535897 & & \\
\hline & & $q_{56}=$ & 0.108057002314816 & & \\
\hline & & $q_{57}=$ & 0.073037987764551 & & \\
\hline & & $q_{58}=$ & -0.015029896410724 & & \\
\hline & & $q_{67}=$ & 0.649000000000000 & & \\
\hline & & $q_{68}=$ & -0.104000000000000 & & \\
\hline & & $q_{78}=$ & 0.755000000000000 & & \\
\hline
\end{tabular}




\section{$\operatorname{SBP}(2,2,8)$}

\begin{tabular}{|c|c|c|c|c|c|}
\hline & $Q$ interior & & $Q$ boundary & & $P$ boundary \\
\hline$a_{1}=$ & 0.820909522170212 & $q_{12}=$ & 0.666853094480032 & $p_{1}=$ & 0.302148955111481 \\
\hline$a_{2}=$ & -0.221666309292960 & $q_{13}=$ & 0.076479756121075 & $p_{2}=$ & 1.517433450301689 \\
\hline$a_{3}=$ & 0.047921361451327 & $q_{14}=$ & -0.606965769217888 & $p_{3}=$ & 0.285932266776763 \\
\hline$a_{4}=$ & -0.005335246984568 & $q_{15}=$ & 0.507594157334931 & $p_{4}=$ & 1.555010218521753 \\
\hline & & $q_{16}=$ & -0.112550043333316 & $p_{5}=$ & 1.012284063361710 \\
\hline & & $q_{17}=$ & -0.055485611063658 & $p_{6}=$ & 0.625467352483611 \\
\hline & & $q_{18}=$ & 0.024074415678823 & $p_{7}=$ & 1.259534176052987 \\
\hline & & $q_{23}=$ & -0.124693288718265 & $p_{8}=$ & 0.942189517390007 \\
\hline & & $q_{24}=$ & 1.849263324563050 & & \\
\hline & & $q_{25}=$ & -1.613862462196115 & & \\
\hline & & $q_{26}=$ & 0.650971333814076 & & \\
\hline & & $q_{27}=$ & -0.083403924642150 & & \\
\hline & & $q_{28}=$ & -0.011421888340565 & & \\
\hline & & $q_{34}=$ & -0.976617502001401 & & \\
\hline & & $q_{35}=$ & 2.003068861125611 & & \\
\hline & & $q_{36}=$ & -1.700521224095258 & & \\
\hline & & $q_{37}=$ & 0.799572166580460 & & \\
\hline & & $q_{38}=$ & -0.173715834206602 & & \\
\hline & & $q_{45}=$ & -0.828851116783346 & & \\
\hline & & $q_{46}=$ & 2.270511280380005 & & \\
\hline & & $q_{47}=$ & -1.645747376085641 & & \\
\hline & & $q_{48}=$ & 0.469767265832744 & & \\
\hline & & $q_{56}=$ & -1.016252629771842 & & \\
\hline & & $q_{57}=$ & 1.584810115859904 & & \\
\hline & & $q_{58}=$ & -0.495272799622414 & & \\
\hline & & $q_{67}=$ & 0.060442326278035 & & \\
\hline & & $q_{68}=$ & -0.010869723751127 & & \\
\hline & & $q_{78}=$ & 0.839267891753151 & & \\
\hline
\end{tabular}




\section{References}

${ }^{1}$ Auld, B., Acounstic Fields and Waves in Solids, Wiley-Interscience, New York, 1973.

${ }^{2}$ Brillouin, L., Wave Propagation in Periodic Structures, Dover, New York, 1953.

${ }^{3}$ Brillouin, L., Wave Propagation and Group Velocity, Academic Press, New York, 1960.

${ }^{4}$ Jenkins, F. and White, H., Fundamentals of Physical Optics, McGraw-Hill, New York, 1937.

${ }^{5}$ Lighthill, J., Waves in Fluids, Cambridge University Press, Cambridge, 1978.

${ }^{6}$ Whitham, G. B., Linear and Nonlinear Waves, Wiley-Interscience, 1974.

${ }^{7}$ Fox-Rabinovitz, M. S., "Computational Dispersion Properties of 3D Staggered Grids for a Nonhydrostatic Anelastic System," Monthly Weather Review, Vol. 124, 1995, pp. 498-510.

${ }^{8}$ Zingg, D. W., "Comparison of high-accuracy finite-difference methods for linear wave propagation," SIAM J. Sci. Comput., Vol. 22, 2000, pp. 476-502.

${ }^{9}$ Kreiss, H.-O. and Scherer, G., "Finite element and finite difference methods for hyperbolic partial differential equations," Aspects of Finite Elements in Partial Differential Equations, Academic Press, Inc., 1974.

${ }^{10}$ Kreiss, H.-O. and Scherer, G., "On the existence of energy estimates for difference approximations of hyperbolic systems," Technical report, Dept. of Scientific Computing, Uppsala University, 1977.

${ }^{11}$ Strand, B., "Summation by Parts for Finite Difference Approximations for d/dx," Journal of Computational Physics, Vol. 110, 1994, pp. 47-67.

${ }^{12}$ Svärd, M. and Nordström, J., "Review of Summation-by-parts schemes for initial-boundary-value problems," Journal of Computational Physics, Vol. 268, 2014, pp. 17-38.

${ }^{13}$ Carpenter, M. H., Nordström, J., and Gottlieb, D., "A Stable and Conservative Interface Treatment of Arbitrary Spatial Accuracy," Journal of Computational Physics, Vol. 148, 1999, pp. 341-365.

${ }^{14}$ Tam, C. and Webb, J., "Dispersion-Reltion-Preserving Finite Difference Schemes for Computational Acoustics," Journal of Computational Physics, Vol. 107, 1993, pp. 262-281.

${ }^{15}$ Johansson, S., "High order difference approximations for the linearized Euler equations," Lic. Uppsala Universitet, Department of Information Technology, 2004.

${ }^{16}$ Linders, V. and Nordström, J., "Uniformly best wavenumber approximations by spatial central difference operators," Journal of Computational Physics, Vol. 300, 2015, pp. 695-709.

${ }^{17}$ Bogey, C. and Bailly, C., "A family of low dispersive and low dissipative explicit schemes for flow and noise computations," Journal of Computational Physics, Vol. 194, 2004, pp. 194-214.

${ }^{18}$ Zingg, D. W., Lomax, H., and Jurgens, H., "High-accuracy finite-difference schemes for linear wave propagation," SIAM J. Sci. Comput., Vol. 17, 1996, pp. 328-346.

${ }^{19}$ Delorme, Y. T., Puri, K., Nordström, J., Linders, V., Dong, S., and Frankel, S. H., "A Simple and Efficient Incompressible Navier-Stokes Solver for Unsteady Complex Geometry Flows on Truncated Domains," Submitted, 2015.

${ }^{20}$ Hashimoto, T., Tanno, I., Yasuda, T., Tanaka, Y., Morinishi, K., and Satofuka, N., "Higher order numerical simulation of unsteady viscous incompressible flows using kinetically reduced local Navier Stokes equations on a GPU," Computers 83 Fluids, Vol. 110, 2015, pp. 108-113.

${ }^{21}$ Minion, M. and Brown, D., "Performance of under-resolved two-dimensional incompressible flow simulations, II," Journal of Computational Physics, Vol. 138, 1997, pp. 734-765.

${ }^{22}$ Clausen, J., "Entropically damped form of artificial compressibility for explicit simulation of incompressible flow," Physical Review E, Vol. 87, 2013, pp. 13309-13321.

${ }^{23}$ Del Rey Fernández, D. C., Hicken, J. E., and Zingg, D. W., "Review of summation-by-parts operators with simultaneous approximation terms for the numerical solution of partial differential equations," Computers \& Fluids, Vol. 95, 1994, pp. 171196.

${ }^{24}$ Carpenter, M. H., Gottlieb, D., and Abarbanel, S., "Time-stable boundary conditions for finite-difference schemes solving hyperbolic systems: Methodology and application to high-order compact schemes," Journal of Computational Physics, Vol. 111(2), 1994.

${ }^{25}$ Carpenter, M. and Gottlieb, D., "Spectral methods on arbitrary grids," Journal of Computational Physics, Vol. 129, 1996, pp. 74-86.

${ }^{26}$ Gassner, G. J., "A skew-symmetric discontinuous Galerkin specral element discretization and its relation to SBP-SAT finite difference methods," SIAM J. Sci. Comput., Vol. 35, 2013, pp. 1233-1253.

${ }^{27}$ Del Rey Fernandez, D. C., Boom, P. D., and Zingg, D., "A generalized framework for nodal first derivative summationby-parts operators," Journal of Computational Physics, Vol. 266, 2014, pp. 214-239.

${ }^{28}$ Nordström, J., "Conservative Finite Difference Formulations, Variable Coefficients, Energy Estimates and Artificial Dissipation," Journal of Scientific Computing, Vol. 29, No. 3, 2006, pp. 375-404.

${ }^{29}$ Boyd, S. and Vandenberghe, L., Convex Optimization, Cambridge University Press, Cambridge, United Kingdom, 7th ed., 2009.

${ }^{30}$ van Rees, W. M., Leonard, A., Pullin, D. I., and Koumoutsakos, P., "A comparison of vortex and pseudo-spectral methods for the simulation of periodic vortical flows at high Reynolds numbers," Journal of Computational Physics, Vol. 230, 2011, pp. 2794-2805.

${ }^{31}$ Hillewaert, K., "3rd international workshop on high order methods," https://www.grc.nasa.gov/hiocfd/, 2015. 